JOURNAL OF SYNCHROTRON RADIATION

ISSN 1600-5775

Received 22 June 2015

Accepted 26 October 2015

Edited by D. A. Reis, SLAC National Accelerator Laboratory, USA

Keywords: XFEL; two color; pump and probe; spectrometer.

\section{Inline spectrometer for shot-by-shot determination of pulse energies of a two-color X-ray free-electron laser}

\author{
Kenji Tamasaku, ${ }^{a *}$ Yuichi Inubushi, ${ }^{b}$ Ichiro Inoue, ${ }^{a, c}$ Kensuke Tono, ${ }^{b}$ \\ Makina Yabashi, and Tetsuya Ishikawa ${ }^{a}$
}

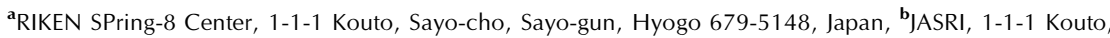
Sayo-cho, Sayo-gun, Hyogo 679-5198, Japan, and ${ }^{\mathbf{C}}$ Graduate School of Frontier Sciences, The University of Tokyo, 5-1-5 Kashiwanoha, Kashiwa, Chiba 277-8561, Japan. *Correspondence e-mail: tamasaku@riken.jp

An inline spectrometer has been developed to monitor shot-by-shot pulse energies of a two-color X-ray beam. A thin film of diamond allows inline operation with minimum absorption. The absolute pulse energy for each color is determined by the inline spectrometer combined with a total pulse-energy monitor. A negative correlation is found between the two-color pulse energies.

\section{Introduction}

Recently, two-color operation (Hara et al., 2013; Lutman et al., 2014) of the X-ray free-electron laser (XFEL) has been realised at SACLA (Ishikawa et al., 2012) and LCLS (Emma et $a l ., 2010)$. These unique sources should open up advanced XFEL applications, such as X-ray pump/X-ray probe experiments. The data analysis requires information about each pulse energy of the two colors. In addition, the pulse energies, which fluctuate shot by shot, must be monitored during the experiment. Since the total pulse-energy monitor assumes a single-color beam (Tono et al., 2013; Emma et al., 2010), a new method is required to measure the spectrally resolved pulse energy. A bent-crystal spectrometer (Zhu et al., 2012) may be used for smaller photon-energy separations, e.g. several $\mathrm{eV}$ as in the case of LCLS. However, there is no method for a keVseparated two-color beam. In this short communication we report absolute and shot-by-shot monitoring of two-color pulse energies at 8.05 and $9.1 \mathrm{keV}$ using a polycrystalline inline spectrometer.

\section{Experimental setup}

Fig. 1(a) shows a schematic diagram of the inline spectrometer. The basic design is the same as for the beamline wavelength monitor (Tono et al., 2013), but is modified to be compact and portable. The two-color beam is intercepted by a thin polycrystalline diamond film. A $15 \mu \mathrm{m}$-thick nanodiamond film was CVD-grown on a silicon substrate, and then the substrate was removed by etching (Tono et al., 2011). The transmittance is calculated to be more than $97 \%$ for photon energies above $8 \mathrm{keV}$ (Henke et al., 1993), enabling inline monitoring. A small part of the beam is diffracted kinematically into different directions according to the Bragg condition. A multi-port CCD (MPCCD) detector (Kameshima et $a l ., 2014)$ captures the diffracted beam image at the repetition rate of SACLA $(30 \mathrm{~Hz})$. The film-to-MPCCD distance is 


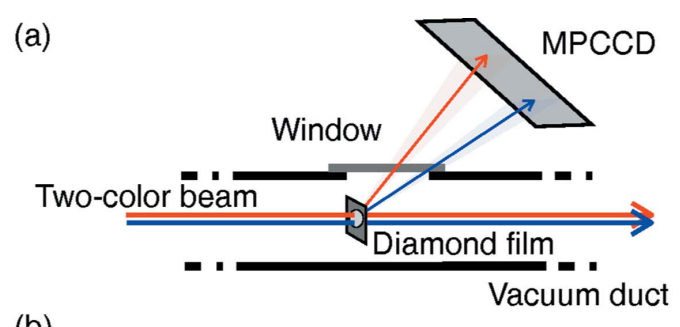

(b)

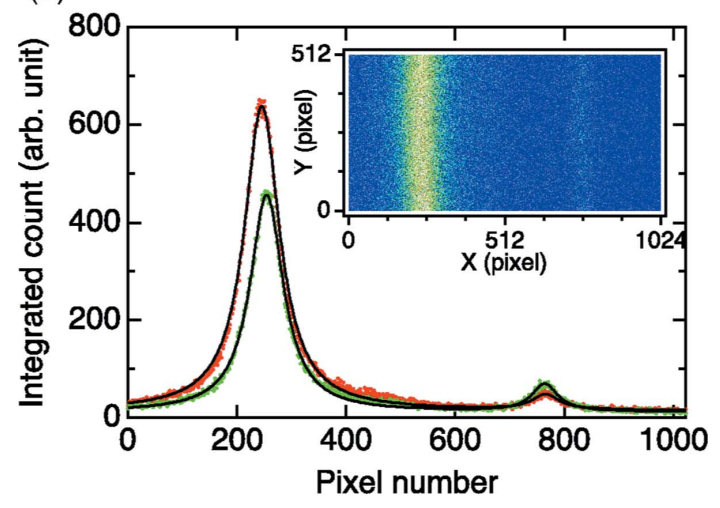

Figure 1

(a) Schematic of the inline spectrometer. (b) Measured single-shot spectra. Inset: a typical diffraction image taken by the MPCCD.

adjustable to obtain a proper separation between the two beam images.

The performance of the inline spectrometer was evaluated at beamline BL3 of SACLA (Ishikawa et al., 2012). The twocolor beam was produced by the so-called split-undulator scheme (Hara et al., 2013). The relative pulse-energy ratio can be tuned by changing the numbers of undulator segments. In this experiment, six upstream segments of the undulator were used for a weak beam at $9.1 \mathrm{keV}$, and the remaining fifteen were used for a strong beam at $8.05 \mathrm{keV}$. The inline spectrometer must be placed downstream of the final aperture, which was a four-jaw slit in this experiment, because the source-toaperture distance, and therefore the solid angle accepted by the aperture, depends on the color in the split-undulator scheme. This is the reason why we have constructed the present portable inline spectrometer.

\section{Result and discussion}

The inset of Fig. 1(b) shows the typical image of the diamond 220 diffraction. The photon energy disperses along the $X$ direction. The $Y$ direction corresponds to the spatial distribution. Strictly speaking, the diffraction image is an arc, which is a part of the Debye-Scherrer cone. However, the effect of the curvature is minor compared with the broad peak width, and is ignored below. The count of each pixel is added vertically to obtain the spectrum. Fig. 1(b) shows spectra obtained in different shots. The peak heights change shot by shot, while the peak photon energies are almost fixed.

The spectrum can be reproduced well by a sum of two Lorentzian functions and a constant,

$$
f(X)=\sum_{i} \frac{A_{i} \Gamma_{i}}{\left(X-X_{0 i}\right)^{2}+\Gamma_{i}^{2}}+B,
$$

where $A$ is the weight, $\Gamma$ is the width and $X_{0}$ is the center pixel of the peak. The constant $B$ represents the background due to air scattering. The peak widths are estimated to be about $150 \mathrm{eV}$. This is larger than the spectral bandwidth of $\sim 50 \mathrm{eV}$ measured separately by the beamline monochromator. The spread is accounted for by the grain size of the diamond film. The width imposes the lower limit of the photon-energy separation between the two colors, which we estimate as about $400 \mathrm{eV}$. Nevertheless, the peak position can be determined with a nominal resolution of $2.1 \mathrm{eV}$, which is given by the pixel size of $50 \mu \mathrm{m}$ and the film-to-MPCCD distance.

The readout of the MPCCD is proportional to both the photon energy and the photon number (Kameshima et al., 2014), so that the fitting parameter, $A$, is proportional to the pulse energy. However, $A_{1}$ and $A_{2}$ cannot be compared without correcting the photon-energy-dependent factors, such as the transmittance of air, $T$, the structure factor, $F$, and the quantum efficiency of the MPCCD, $Q$. Correcting these factors, the absolute pulse energy may be given by

$$
P_{i}=C \frac{A_{i}}{T_{i}\left|F_{i}\right|^{2} Q_{i}},
$$

where $i=1,2$ denotes the color, and $C$ is a common conversion constant. The relative pulse-energy variation of each color can be determined without knowledge of $C$. The structure factor appears as $\left|F_{i}\right|^{2}$ because of the kinematical nature of diffraction.

If we performed an additional measurement in the singlecolor mode, we could determine $C$ directly by a total pulseenergy monitor. Here, we discuss another approach without changing the color mode. In general, the sensitivity of the total pulse-energy monitor depends on the photon energy. When a pulse-energy monitor measures the two-color beam, the output signal, $S$, may be given by a sum of that for each color:

$$
S=P_{1} / D_{1}+P_{2} / D_{2}
$$

Here, $D$ is the known conversion coefficient of the total pulseenergy monitor. Combining (3) with (2), $C$ can be determined from $A_{1,2}$ and $S$, which are to be measured experimentally by the inline spectrometer and the total pulse-energy monitor, respectively.

In the present experiment, we used a beam-position monitor (BPM) as the total pulse-energy monitor (Tono et al., 2013). The BPM consists of a thin diamond film and quadrant photodiodes, which measure the backscattered X-rays by the film. The pulse energy is determined from $S$, the sum of the four charges measured by the photodiodes. The output of the photodiode is not the current but the charge, because the XFEL is a pulsed source. The conversion coefficient of the BPM, $D$, is calibrated to a cryogenic radiometer (Kato et al., 2012).

Fig. 2(a) shows the relation between the measured $S$ by the BPM and the calculated $S$ from $A_{1,2}$ using (2) and (3). The data points lie in the vicinity of a line crossing the origin, 

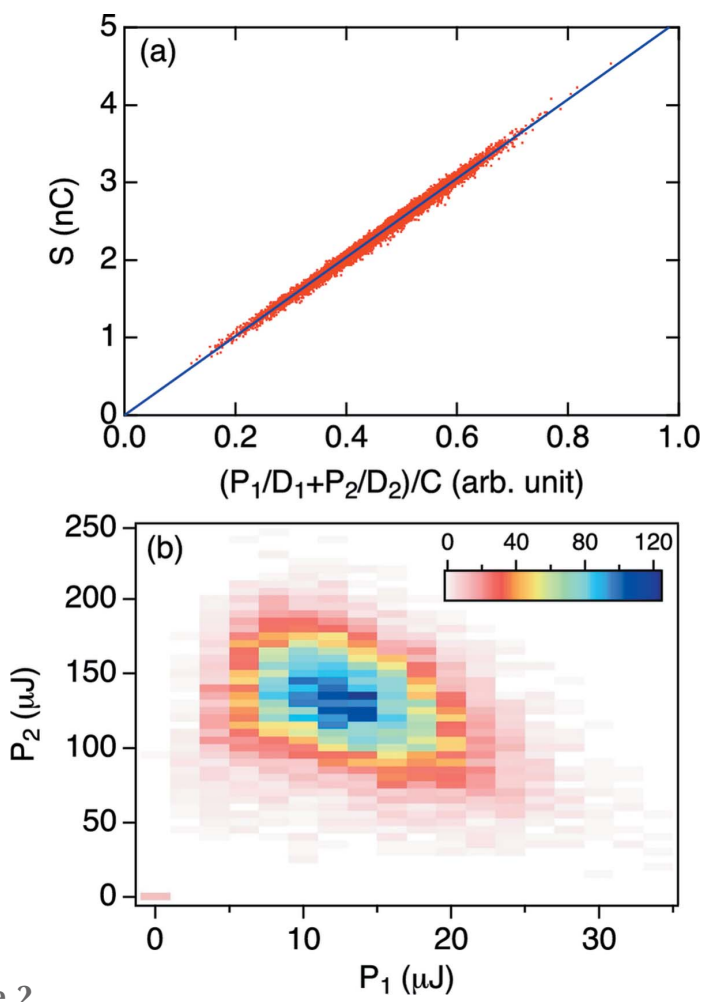

Figure 2

(a) Measured output signal of the total pulse-energy monitor (BPM) plotted as a function of the calculated one from the spectrum analysis of the inline spectrometer. The solid line represents the best fitting with a linear function. (b) Distribution of two-color pulse energies, $\left(P_{1}, P_{2}\right)$. The color bar indicates the frequency.

indicating the validity of our analysis. The conversion coefficient is estimated to be $C=5.0883 \pm 0.00162$ from the slope of the linear fitting. Now, the shot-by-shot pulse energy for each color can be calculated using (2). The $\left(P_{1}, P_{2}\right)$ distribution of 10000 shots is plotted in Fig. 2(b). A negative correlation is found between $P_{1}$ and $P_{2}$, which is considered to be a natural consequence of the fact that the two colors originate from the same electron beam.

Finally, we discuss the error of $P_{1,2}$. The error arises from the fitting parameters, $C$ and $A_{1,2}$, and the conversion coefficients, $D_{1,2}$. The average photon numbers at the peak are 4.2 photons per pixel for $8.05 \mathrm{keV}$ and 0.25 for $9.1 \mathrm{keV}$. The shot noise dominates the fitting error of $A_{1,2}$, while the readout noise of the MPCCD is negligible. The uncertainty of $A_{1}$ and $A_{2}$ are estimated to be $3.1 \%$ and $7.5 \%$, respectively. The larger error for $9.1 \mathrm{keV}$ is due to the weaker signal. The relative uncertainty of $P_{1,2}$ is determined by that for $A_{1,2}$, because the error of $C$ is much smaller. The uncertainty of the absolute pulse energy depends on that of the total pulse-energy monitor as well. When we adapt $2.5 \%$ evaluated at $9.6 \mathrm{keV}$ to that of $D_{1,2}$ (Kato et al., 2012), we estimate the uncertainty of $P_{1,2}$ to be $4.0 \%$ for $8.05 \mathrm{keV}$ and $8.0 \%$ for $9.1 \mathrm{keV}$.

\section{Conclusion}

In conclusion, we have developed and operated successfully an inline spectrometer to monitor the relative two-color pulse energies of SACLA shot-by-shot. Furthermore, using a calibrated BPM, the absolute two-color pulse energies are determined, which will serve for quantitative analysis of twocolor XFEL experiments. Although the photon-energy resolution of the present inline spectrometer is not enough for smaller separations, it can be improved by increasing the grain size of the diamond film.

\section{Acknowledgements}

The experiments were performed with the approval of JASRI (proposal No. 2014A8004).

\section{References}

Emma, P. et al. (2010). Nat. Photon. 4, 641-647.

Hara, T., Inubushi, Y., Katayama, T., Sato, T., Tanaka, H., Tanaka, T., Togashi, T., Togawa, K., Tono, K., Yabashi, M. \& Ishikawa, T. (2013). Nat. Commun. 4, 2919.

Henke, B. L., Gullikson, E. M. \& Davis, J. C. (1993). At. Data Nucl. Data Tables, 54, 181-342.

Ishikawa, T. et al. (2012). Nat. Photon. 6, 540-544.

Kameshima, T., Ono, S., Kudo, T., Ozaki, K., Kirihara, Y., Kobayashi, K., Inubushi, Y., Yabashi, M., Horigome, T., Holland, A., Holland, K., Burt, D., Murao, H. \& Hatsui, T. (2014). Rev. Sci. Instrum. 85, 033110.

Kato, M., Tanaka, T., Kurosawa, T., Saito, N., Richter, M., Sorokin, A. A., Tiedtke, K., Kudo, T., Tono, K., Yabashi, M. \& Ishikawa, T. (2012). Appl. Phys. Lett. 101, 023503.

Lutman, A. A., Decker, F.-J., Arthur, J., Chollet, M., Feng, Y., Hastings, J., Huang, Z., Lemke, H., Nuhn, H.-D., Marinelli, A., Turner, J. L., Wakatsuki, S., Welch, J. \& Zhu, D. (2014). Phys. Rev. Lett. 113, 254801.

Tono, K., Kudo, T., Yabashi, M., Tachibana, T., Feng, Y., Fritz, D., Hastings, J. \& Ishikawa, T. (2011). Rev. Sci. Instrum. 82, 023108.

Tono, K., Togashi, T., Inubushi, Y., Sato, T., Katayama, T., Ogawa, K., Ohashi, H., Kimura, H., Takahashi, S., Takeshita, K., Tomizawa, H., Goto, S., Ishikawa, T. \& Yabashi, M. (2013). New J. Phys. 15, 083035 .

Zhu, D., Cammarata, M., Feldkamp, J. M., Fritz, D. M., Hastings, J. B., Lee, S., Lemke, H. T., Robert, A., Turner, J. L. \& Feng, Y. (2012). Appl. Phys. Lett. 101, 034103. 